\title{
Relação entre toxicidade de proteínas Vip3Aa e sua capacidade de ligação a receptores intestinais de lepidópteros-praga
}

\author{
Suzana Cristina Marucci(1), Camila Soares Figueiredo(1), Renata Izabel Dozzi Tezza(2), \\ Eliane Cristina da Cunha Alves ${ }^{(1)}$, Manoel Victor Franco Lemos ${ }^{(1)}$ e Janete Apparecida Desidério(1)
}

(1)Universidade Estadual Paulista (Unesp), Faculdade de Ciências Agrárias e Veterinárias, Departamento de Biologia Aplicada à Agropecuária, Rodovia Prof. Paulo Donato Castellane, s/non, Zona Rural, CEP 14884-900 Jaboticabal, SP, Brasil. E-mail: suzy_marucci@yahoo.com.br, camila_sfigueiredo@hotmail.com, elianea@fcav.unesp.br, mvictor@fcav.unesp.br, janete@fcav.unesp.br (2)Unesp, Centro de Recursos Biológicos e Biologia Genômica, CEP 14884-900 Jaboticabal, SP, Brasil. E-mail: renata25@fcav.unesp.br

Resumo - O objetivo deste trabalho foi avaliar a toxicidade de novas proteínas Vip3Aa e sua capacidade de ligação a vesículas de membrana da microvilosidade apical (VMMA) do intestino de lagartas neonatas de Spodoptera frugiperda, Anticarsia gemmatalis e Heliothis virescens. Proteínas expressas pelos genes vip3Aa42 e vip3Aa43 mostraram-se tóxicas a S. frugiperda $\left(\mathrm{CL}_{50} \mathrm{de} 78,2 \mathrm{e} 113 \mathrm{ng} \mathrm{cm}^{-2}\right.$, respectivamente) e A. gemmatalis $\left(\mathrm{CL}_{50}\right.$ de 239,2 e $57,5 \mathrm{ng} \mathrm{cm}^{-2}$, respectivamente), e pouco tóxicas a $H$. virescens $\left(\mathrm{CL}_{50}>5.000 \mathrm{ng} \mathrm{cm}^{-2}\right)$. Os ensaios de ligação às VMMA mostraram que as proteínas unem-se de forma efetiva aos receptores nas vesículas das espécies avaliadas, mas essa capacidade de ligação somente é efetiva na ativação da toxicidade para as populações avaliadas de $S$. frugiperda e A. gemmatalis.

Termos para indexação: Anticarsia gemmatalis, Heliothis virescens, Spodoptera frugiperda, manejo de resistência, proteína Cry, vesículas de membrana.

\section{Relationship between toxicity of Vip3Aa proteins and their binding capacity to intestine receptors of lepidopteran pests}

\begin{abstract}
The objective of this work was to evaluate the toxicity of new Vip3Aa proteins and their binding capacity to brush-border membrane vesicles (BBMV) in the intestine of Spodoptera frugiperda, Anticarsia gemmatalis, and Heliothis virescens neonate larvae. The proteins expressed by the genes vip3Aa42 and vip3Aa43 showed toxicity to $S$. frugiperda $\left(\mathrm{LC}_{50}\right.$ of 78.2 and $113 \mathrm{ng} \mathrm{cm}^{-2}$, respectively) and A. gemmatalis $\left(\mathrm{LC}_{50}\right.$ of 239.2 and $57.5 \mathrm{ng} \mathrm{cm}^{-2}$, respectively), but they showed low toxicity to $H$. virescens $\left(\operatorname{LC}_{50}>5,000 \mathrm{ng} \mathrm{cm}^{-2}\right)$. BBMV binding assays showed that the proteins bind effectively to the receptors on vesicles of the evaluated species, but this binding capacity is only effective on the activation of toxicity to the evaluated populations of S. frugiperda and A. gemmatalis.
\end{abstract}

Index terms: Anticarsia gemmatalis, Heliothis virescens, Spodoptera frugiperda, management of resistance, Cry proteins, membrane vesicles.

\section{Introdução}

A bactéria Bacillus thuringiensis tem sido utilizada na proteção das lavouras (Raymond et al., 2010) como alternativa ao uso de agrotóxicos. Ela é encontrada naturalmente no solo e, durante sua fase de esporulação, produz diferentes $\delta$-endotoxinas, ou proteínas Cry, tóxicas a insetos de diversas ordens, inclusive lepidópteros, dípteros e coleópteros (Van Frankenhuyzen, 2009).

Embora ainda haja debate, de modo geral, aceita-se que as proteínas Cry são convertidas em fragmentos tóxicos pela ação de enzimas digestivas nos insetos suscetíveis e que, posteriormente, elas se ligam a receptores específicos na borda da membrana epitelial no intestino, o que gera lesões capazes de destruir as células e matar o inseto (Vachon et al., 2012).

As proteínas Cry são eficientes no controle de insetos-praga; no entanto, estudos recentes sugerem que muitas pragas tenham evoluído com resistência a estas toxinas, como: Helicoverpa zea (Boddie, 1850) (Lepidoptera: Noctuidae), que tem apresentado resistência à proteína Cry1Ac expressa em algodão, nos EUA (Tabashnik et al., 2008); Spodoptera frugiperda (J.E. Smith, 1797) (Lepidoptera: Noctuidae), com resistência à proteína Cry1F expressa 
em milho, em Porto Rico e no Brasil (Storer et al., 2010); e Pectinophora gossypiella (Saunders, 1844) (Lepidoptera: Gelechiidae), resistente à proteína Cry1Ac expressa em algodão, na Índia (Duhrua \& Gujar, 2011). Esses casos de evolução de resistência geram grande preocupação, uma vez que colocam em risco uma tecnologia recente e promissora, como a do uso de plantas Bt (Ferré \& Van Rie, 2002; Tabashnik et al., 2008).

As proteínas Vip3Aa, também produzidas pela bactéria $B$. thuringiensis, sãouma alternativa promissora no manejo da resistência, em razão de sua toxicidade a insetos da ordem Lepidoptera, inclusive Agrotis ipsilon (Hufnagel, 1766) (Lepidoptera: Noctuidae), Spodoptera exigua (Hübner, 1808) (Lepidoptera: Noctuidae), S. frugiperda, Heliothis virescens (Fabricius, 1777) (Lepidoptera: Noctuidae) e H. zea, alguns dos quais com baixa suscetibilidade à proteína Cry (Estruch et al., 1996).

Pouco se sabe quanto ao modo de ação das proteínas Vip, além de que elas atuam por meio da formação de poros nas células epiteliais do intestino médio. Essas proteínas são ingeridas como protoxinas e processadas em toxinas pelas proteases do intestino do inseto, que se ligam a receptores específicos e causam a lise das células do intestino médio (Lee et al., 2003).

A interação proteína-receptor é necessária para que ocorra a toxicidade das proteínas (Hofmann et al., 1988), embora por si só isto não seja suficiente (Wolfersberger, 1990). Há evidências de que as proteínas Vip3Aa ligam-se a receptores diferentes dos das proteínas Cry1, na membrana epitelial do intestino médio dos insetos-praga (Sena et al., 2009; Hamadou-Charfi et al., 2013). Esta é uma informação relevante para o manejo da resistência, uma vez que alterações que diminuem a ligação das proteínas com os receptores estão associadas a elevados níveis de resistência às toxinas Cry (Ferré \& Van Rie, 2002). As diferentes propriedades de ligação entre proteínas Vip e Cry sugerem que elas podem ser usadas em conjunto ou em rotação no controle de pragas. Nesse sentido, é importante a análise de toxicidade e ligação de diferentes proteínas Vip3Aa, em diferentes espécies de lepidópteros.

O objetivo deste trabalho foi avaliar a toxicidade de novas proteínas Vip3Aa e sua capacidade de ligação a vesículas de membrana da microvilosidade apical (VMMA) do intestino de larvas neonatas de S. frugiperda, A. gemmatalis e $H$. virescens.

\section{Material e Métodos}

As linhagens bacterianas de B. thuringiensis var. tolworthi $\mathrm{HD} 125$ e $B$. thuringiensis var. kurstaki HD1, usadas no presente estudo, provieram do Bacillus Genetic Stock Center, Columbus, Ohio (EUA). Essas linhagens são mantidas no Laboratório de Genética de Bactérias e Biotecnologia Aplicada (LGBBA), da Unesp, em Jaboticabal, SP. A primeira linhagem é considerada, atualmente, um padrão para o gene vip $3 \mathrm{Aa}$, além de possuir também o gene vip $3 A a 8$ - acesso número AF399667 GenBank (2014b), identificado por Loguercio et al. (2002). A segunda linhagem é considerada padrão para vários genes crylA e cry2, além de também possuir o gene vip $3 A a 33$ - acesso número GU073128 GenBank (2014a), cuja sequência foi submetida por Sauka et al. (2012).

As linhagens de Bt foram cultivadas em placas de Petri, em meio de cultura "brain heart infusion", BHI, (HiMedia, Mumbai, India) que continha $200 \mathrm{~g}$ de infusão de cérebro de bezerro, $10 \mathrm{~g}$ de protease peptona, $5 \mathrm{~g}$ de cloreto de sódio, $250 \mathrm{~g}$ de infusão de coração bovino, $2 \mathrm{~g}$ de dextrose, $2,5 \mathrm{~g}$ de fosfato dissódico, $\mathrm{H}_{2} \mathrm{O}$ q.s.p $1.000 \mathrm{~mL}, \mathrm{pH} 7,4 \pm 0,2$, adicionado de ágar, a $30^{\circ} \mathrm{C}$, por 16 horas. As colônias isoladas foram inoculadas em $2 \mathrm{~mL}$ de meio BHI líquido e mantidas a $30^{\circ} \mathrm{C}$, sob agitação de $200 \mathrm{rpm}$ por 16 horas.

O DNA das linhagens foi extraído pelo método de fervura (Letowski et al., 2005) e submetido à amplificação do gene vip3Aa com os oligonucleotídeos Vip5 e Vip6, descritos por Loguercio et al. (2002) (Tabela 1). A reação em cadeia da polimerase (PCR) foi realizada em volume total de $25 \mu \mathrm{L}$, com: $150 \mathrm{ng}$ de DNA; 1 U de enzima Platinum Taq DNA Polymerase High Fidelity (Invitrogen, Life Technologies do Brasil, São Paulo, SP), 1 X High Fidelity PCR Buffer (Invitrogen); $1,5 \mathrm{mmol} \mathrm{L} \mathrm{L}^{-1}$ de $\mathrm{MgSO}_{4}, 0,5 \mathrm{pmol}$ de cada oligonucleotídeo; $0,16 \mathrm{mmol} \mathrm{L}^{-1}$ de dNTP, e água destilada deionizada estéril (q.s.p. $25 \mu \mathrm{L}$ ). Utilizaram-se as seguintes temperaturas e tempos de incubação: $94^{\circ} \mathrm{C}, 2 \mathrm{~min} ; 30$ ciclos de $94^{\circ} \mathrm{C}, 1 \mathrm{~min}$; $53^{\circ} \mathrm{C}, 1 \mathrm{~min} ; 68^{\circ} \mathrm{C}, 2 \mathrm{~min} ; 68^{\circ} \mathrm{C}, 5 \mathrm{~min}$; e $4^{\circ} \mathrm{C}$, até a utilização da amostra.

Os genes vip3Aa completos foram inseridos no vetor pGEM-T Easy (Promega, Madison, WI, EUA), segundo 
instruções do fabricante. Células de Escherichia coli $\mathrm{DH} 10 \mathrm{~B}$ foram usadas para a transformação por choque térmico e selecionadas em placas com meio LB-ágar, suplementado com ampicilina a $100 \mu \mathrm{g} \mathrm{mL}^{-1}$ (Sambrook \& Russell, 2001). Os clones transformantes foram selecionados e confirmados por PCR, por meio da amplificação do fragmento de interesse com os oligonucleotídeos T7 e SP6 do vetor de clonagem.

Os clones foram sequenciados com o kit BigDye Terminator v3.1 Cycle Sequencing (Applied Biosystems, Foster City, CA, EUA), em sequenciador automático ABI 3100 (Applied Biosystems). Para obtenção da sequência completa do gene, utilizou-se a estratégia "primer walking", com anelamento de iniciadores específicos ao longo do gene (Tabela 1).

A sequência consenso do gene foi gerada com uso das ferramentas Phred/Phrap/Consed (Ewing \& Green, 1998; Ewing et al., 1998; Gordon et al., 1998) e comparada à de outros genes vip $3 A$, por meio do programa "basic local alignment search tool" (BLAST), por meio do algoritmo BLASTx, a partir do banco de dados do National Center for Biotechnology Information (NCBI). As sequências foram enviadas ao Comitê Bacillus thuringiensis Toxin Gene Nomenclature (Crickmore et al., 2014) para a classificação dos genes.

Para avaliação da expressão heteróloga, os genes vip3Aa foram subclonados em vetor pET SUMO "Champion pET SUMO Expression System" (Invitrogen), de acordo com as recomendações do fabricante. Células de E. coli BL21(DE3) One Shot (Invitrogen) foram utilizadas para a transformação, pelo método de choque térmico, e selecionadas em LB, com canamicina $\left(50 \mu \mathrm{g} \mathrm{mL}^{-1}\right)$. Os clones transformantes foram selecionados e confirmados por PCR pela amplificação dos genes de interesse.

Para a indução dos genes vip $3 A a$, realizou-se o cultivo dos clones em LB-ágar suplementado com canamicina $\left(50 \mu \mathrm{g} \mathrm{mL}^{-1}\right)$. Para isso, utilizaram-se $12 \mu \mathrm{L}$ da cultura dos clones, em células de E. coli BL21 (DE3) One Shot, assepticamente estocadas em glicerol (30\%) estéril, e armazenadas em ultra-freezer a $-80^{\circ} \mathrm{C}$. As placas foram incubadas a $37^{\circ} \mathrm{C}$ por 16 horas. Em seguida, preparou-se um pré-inóculo, em $10 \mathrm{~mL}$ de meio LB e canamicina $\left(50 \mu \mathrm{g} \mathrm{mL}^{-1}\right)$, a partir de uma colônia isolada do clone, e a cultura foi incubada a $37^{\circ} \mathrm{C}$ e agitada a $250 \mathrm{rpm}$, por 16 horas.

Após esse período, $4 \mathrm{~mL}$ do pré-inóculo foram transferidos para $400 \mathrm{~mL}$ de $\mathrm{LB}$ com canamicina $\left(50 \mu \mathrm{g} \mathrm{mL}^{-1}\right)$, e a cultura foi incubada a $37^{\circ} \mathrm{C}$ sob agitação de $250 \mathrm{rpm}$, até que a densidade óptica a $600 \mathrm{~nm}$ estivesse entre 0,5 e 0,8. Em seguida, as culturas foram induzidas pela adição de IPTG (isopro pil- $\beta$-D-1-tiogalactopiranosídeo), à concentração final de $0,4 \mathrm{mmol} \mathrm{L}^{-1}$, e mantidas a $22^{\circ} \mathrm{C}$ sob agitação de 200 rpm por 5 horas.

Para extração das proteínas, as células foram coletadas por centrifugação, por $30 \mathrm{~min}$, a $17.400 \mathrm{~g}$, e os sedimentos obtidos ("pellets") foram mantidos em gelo. Para o início da lise celular, adicionaram-se $20 \mathrm{~mL}$ de tampão de lise $\left(50 \mathrm{mmol} \mathrm{L}^{-1}\right.$ fosfato de

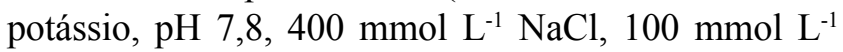
$\mathrm{KCl}, 10 \%$ glicerol, $0,5 \%$ Triton $\mathrm{X}-100$, e $10 \mathrm{mmol} \mathrm{L}^{-1}$ imidazol) aos sedimentos bacterianos, que foram então ressuspendidos por agitação vigorosa em aparelho do tipo "vortex". Adicionaram-se $600 \mu \mathrm{L}$ de lisozima (100 $\left.\mathrm{mg} \mathrm{mL}^{-1}\right), 200 \mu \mathrm{L}$ de DNAse I $\left(1 \mathrm{mg} \mathrm{mL}^{-1}\right)$ e $20 \mu \mathrm{L}$ de PMSF (fenilmetanosulfonilfluoreto) a $0,1 \mathrm{~mol} \mathrm{~L}^{-1} \mathrm{e}$,

Tabela 1. Oligonucleotídeos iniciadores utilizados para amplificação e sequenciamento dos genes vip3Aa das linhagens de Bacillus thuringiensis var. tolworthi HD125 e Bacillus thuringiensis var. kurstaki HD1.

\begin{tabular}{|c|c|c|}
\hline Iniciador $^{(1)}$ & Sequência( ${ }^{(2)}$ & Posição nos genes \\
\hline Vip5 & $\begin{array}{l}\text { 5'ATGAACAAGAATAATACTAAATTAAGC 3' (d) } \\
\end{array}$ & $1-27$ \\
\hline Vip6 & 5' GATCTTACTTAATAGAGACAT 3' (r) & $2.370-2.353$ \\
\hline A1 & 5'CTCTATGTTGAGTGATGTAATG 3'(d) & $367-388$ \\
\hline A2 & 5’ TCCGACCTCACTGCCACTTG 3’ (r) & $808-789$ \\
\hline A3 & 5, ССТСССТАСАСТТТСТААТАС (d) & 967-987 \\
\hline A4 & 5' TAAGTGCTAATGATGATGG 3' (d) & $1.401-1.419$ \\
\hline A5 & 5' CTGGGCACAATAATTTAT (r) & $2.000-2.018$ \\
\hline A6 & 5' TGATCCCGTACTCGTCC 3' (r) & $2.070-2.054$ \\
\hline
\end{tabular}

(1)Iniciadores Vip5 e Vip6, obtidos de Loguercio et al. (2002); e iniciadores A1-A6, obtidos no presente trabalho. (2)Códons ATG e TTA sublinhados representam o códon de início e o códon de terminação, respectivamente; d, direto; r, reverso. 
em seguida, as amostras foram incubadas sob agitação suave, a $37^{\circ} \mathrm{C}$, por $30 \mathrm{~min}$.

Para o total rompimento das células, as amostras foram mantidas em gelo e submetidas à sonicação, com intensidade média, por $60 \mathrm{~s}$ e intervalo de $15 \mathrm{~s}$, tendose repetido o procedimento por três vezes. Em seguida, o material foi centrifugado a $17.400 \mathrm{~g}$ por $30 \mathrm{~min}$, a $4^{\circ} \mathrm{C}$, e o sobrenadante foi coletado. A expressão das proteínas foi analisada em gel de poliacrilamida-SDS 12\%, conforme Laemmli (1970), e a concentração foi determinada por densitometria, tendo-se utilizado o programa ImageQuant TL 8.1 (GE Healthcare Bio-Sciences AB, Uppsala, Suécia), com soroalbumina bovina (BSA) como padrão. O lisado proteico foi usado nos bioensaios e na posterior purificação das proteínas Vip3Aa.

Nos bioensaios com as neonatas de $A$. gemmatalis, $S$. frugiperda e $H$. virescens, a estimativa das concentrações letais de 50 e $90 \%\left(\mathrm{CL}_{50}\right.$ e $\left.\mathrm{CL}_{90}\right)$ foi feita com diferentes concentrações das protoxinas Vip3Aa42 e Vip3Aa43, diluídas em tampão de lise. Utilizaram-se as seguintes concentrações: 6,25, 12,5, 25, 50, 100, 200 e $400 \mathrm{ng} \mathrm{cm}^{-2}$ para $S$. frugiperda e $A$. gemmatalis. Para $H$. virescens, utilizaram-se as concentrações de 100, 500, 1.500, 2.000, $5.000 \mathrm{e}$ $10.000 \mathrm{ng} \mathrm{cm}^{-2}$. Essas concentrações foram definidas em bioensaios preliminares.

Para a criação das espécies avaliadas, utilizou-se a dieta artificial proposta por Greene et al. (1976). A dieta foi individualizada nas células de placas de poliestireno, com 128 poços de $2 \mathrm{~cm}^{2}$, tendo-se utilizado $1 \mathrm{~mL}$ de dieta por poço (Bio-serv, Frenchtown, NJ, USA).

Os bioensaios consistiram de tratamentos da superfície da dieta com as proteínas Vip3Aa42 ou Vip3Aa43. Após a completa secagem da dieta, adicionaram-se $50 \mu \mathrm{L}$ do lisado proteico em cada poço da placa. Dois controles negativos foram utilizados: dieta artificial tratada com $50 \mu \mathrm{L}$ de extrato proteico de E. coli BL21 (DE3) One Shot; e dieta artificial tratada com $50 \mu \mathrm{L}$ de tampão de lise.

As placas foram mantidas em fluxo laminar e, após a absorção do lisado proteico pela dieta, uma neonata foi infestada em cada célula com o auxílio de um pincel fino. As placas foram seladas com plásticos auto-adesivos (Bio-serv, Frenchtown, NJ, USA) e transferidas para uma sala climatizada a $26 \pm 2^{\circ} \mathrm{C}$, com umidade relativa do ar de $70 \pm 10 \%$ e fotoperíodo de 14:10 horas (luz: escuro) (Marçon et al., 1999).
Utilizou-se o delineamento experimental inteiramente casualizado, com quatro repetições por concentração e 16 neonatas por repetição. A mortalidade foi avaliada

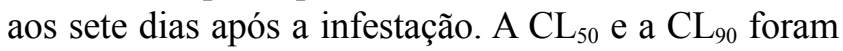
calculadas por meio da análise de Probit (Finney, 1971), com uso do programa de estatística POLO-PC (LeOra Software, Berkeley, CA).

As proteínas Vip3Aa presentes nos lisados de $E$. coli foram purificadas em colunas His-Trap HP (GE Healthcare UK Ltd., Little Chalfont, Buckinghamshire, Inglaterra), previamente carregadas com $\mathrm{Ni}^{2+}$. As colunas apresentam afinidade pela cauda de histidina (6xHis) presente nas proteínas recombinantes. Inicialmente, a coluna foi equilibrada com $20 \mathrm{mmol}$ $\mathrm{L}^{-1}$ de tampão fosfato de sódio, $\mathrm{pH} 7,4,0,5 \mathrm{~mol} \mathrm{~L}^{-1}$ de $\mathrm{NaCl}$ e $50 \mathrm{mmol} \mathrm{L}^{-1}$ de imidazol. O lisado proteico foi eluído com o mesmo tampão fosfato de sódio, com $300 \mathrm{mmol} \mathrm{L}^{-1}$ de imidazol. Frações de $1 \mathrm{~mL}$ foram coletadas em microtubos e analisadas em gel de poliacrilamida-SDS $12 \%$.

A concentração das proteínas purificadas foi determinada pelo método de Bradford (Bradford, 1976), tendo-se utilizado soroalbumina bovina (BSA) como padrão. As proteínas purificadas foram ativadas por proteólise, com uso da tripsina pancreática bovina (Sigma-Aldrich Corp., St. Louis, MO, EUA), à proporção de 1:10 (tripsina:protoxina), e incubadas sob agitação de $140 \mathrm{rpm}$, a $37^{\circ} \mathrm{C}$ por uma hora e 30 minutos. Após a incubação, as amostras foram centrifugadas a $13.000 \mathrm{~g}$ por $15 \mathrm{~min}$, e os sobrenadantes foram analisados em gel de poliacrilamida-SDS $12 \%$, para verificar a eficiência da digestão.

Para os ensaios de proteólise com suco intestinal, larvas de último instar das três espécies estudadas, mantidas em dieta artificial, foram imobilizadas em gelo por 5 min e dissecadas longitudinalmente para coletar a membrana peritrófica, juntamente com o bolo alimentar. Para cada amostra, cinco membranas peritróficas foram colocadas em microtubos, centrifugadas a $13.000 \mathrm{~g}$ por $20 \mathrm{~min}$, e o sobrenadante foi coletado. A concentração proteica do suco intestinal foi obtida pelo método de Bradford, tendo-se utilizado uma proporção de 1:10 de suco intestinal:protoxina para a proteólise. A mistura foi incubada sob agitação de $140 \mathrm{rpm}$, a $37^{\circ} \mathrm{C}$ por uma hora e 30 minutos e, após a incubação, as amostras foram centrifugadas a $13.000 \mathrm{~g}$ por $15 \mathrm{~min}$; os sobrenadantes foram analisados em gel de poliacrilamida-SDS $12 \%$. 
As toxinas purificadas e ativadas com tripsina foram quantificadas pelo método de Bradford e marcadas com biotina Amersham ECL Protein Biotinylation Module (GE Healthcare UK Ltd., Little Chalfont, Buckinghamshire, Inglaterra). Adicionaram-se $40 \mu \mathrm{L}$ de biotina para cada $1 \mathrm{mg}$ de proteína. A mistura foi incubada sob agitação constante, em temperatura ambiente por uma hora. A proteína biotinilada foi, então, purificada em colunas PD10-Desalting (GE Healthcare Bio-Sciences AB, Uppsala, Suécia) e eluída com tampão PBS, pH 7,4.

Frações coletadas foram aplicadas em gel de poliacrilamida-SDS $9 \%$ e, posteriormente, eletrotransferidas para membrana de nitrocelulose Amersham Hybond ECL Nitrocellulose Membrane (GE Healthcare UK Ltd., Little Chalfont, Buckinghamshire, Inglaterra). A membrana foi bloqueada com 5\% de leite em pó desnatado por uma hora e, então, incubada com Streptavidin-AP Conjugate (Roche Molecular Systems Inc., Pleasanton, CA, EUA) na diluição de 1:2.000 por uma hora. A revelação foi realizada com o substrato BCIP/NBT Liquid Substrate System (Sigma-Aldrich Corp., St. Louis, MO, EUA).

Larvas de ultimo instar, alimentadas em dieta artificial, foram imobilizadas em gelo por $5 \mathrm{~min}$ e dissecadas longitudinalmente. Os intestinos médios (1 mg) foram lavados em tampão MET (250 mmol L-1 manitol, $17 \mathrm{mmol} \mathrm{L}^{-1}$ Tris-HCl, $5 \mathrm{mmol} \mathrm{L}{ }^{-1}$ EGTA, $\mathrm{pH} 7,5)$. As vesículas de membrana da microvilosidade apical (VMMA) foram preparadas pelo método de precipitação diferencial de magnésio (Wolfersberger et al., 1987) e quantificadas pelo método de Bradford.

Os ensaios de ligação das proteínas às VMMAs foram realizados conforme Abdelkefi-Mesrati et al. (2011), com modificações, e caracterizaram-se pela ligação das proteínas Vip3Aa às VMMAs extraídas do intestino médio de $S$. frugiperda, A. gemmatalis e H. virescens.

Cada proteína biotinilada (100 ng) foi incubada com $10 \mu \mathrm{g}$ de VMMAs, em $100 \mu \mathrm{L}$ de tampão PBS $(\mathrm{pH} 7,4), \mathrm{a} 28^{\circ} \mathrm{C}$ por uma hora. As proteínas não ligadas às VMMAs foram removidas por centrifugação a $13.000 \mathrm{~g}$, por $15 \mathrm{~min}$. As VMMAs foram lavadas duas vezes em PBS, e ressuspendidas em $20 \mu \mathrm{L}$ de PBS e $10 \mu \mathrm{L}$ de tampão de amostra $\left(0,125 \mathrm{~mol} \mathrm{~L}^{-1}\right.$ de Tris, pH $6,8,4 \%$ de SDS, $0,004 \%$ de azul de bromofenol, $20 \%$ de glicerol e $10 \%$ de $\beta$-mercaptoetanol).
Em seguida, as amostras foram fervidas por $5 \mathrm{~min}$ e aplicadas em gel de poliacrilamida-SDS 9\%.

A transferência das proteínas para a membrana de nitrocelulose e a imunodetecção foram realizadas conforme já descrito (marcação das proteínas ativadas com biotina).

O ensaio de competição homóloga das proteínas pelas VMMAs foi realizado conforme Abdelkefi-Mesrati et al. (2011), com modificações, e caracterizou-se pela ligação e competição das proteínas Vip3Aa aos receptores extraídos do intestino médio de $S$. frugiperda, A. gemmatalis e $H$. virescens.

A proteína biotinilada (100 ng) foi incubada com $10 \mu \mathrm{g}$ de VMMAs, em $100 \mu \mathrm{L}$ de tampão PBS (pH 7,4), a $28^{\circ} \mathrm{C}$ por uma hora, na presença da mesma proteína tripsinizada não marcada com biotina, em excesso de $500,1.000$ e 2.000 vezes.

As proteínas não ligadas à VMMAs foram removidas e analisadas da mesma forma descrita acima. A transferência das proteínas para a membrana de nitrocelulose e a imunodetecção também foram realizadas conforme descrição prévia (marcação das proteínas ativadas com biotina).

\section{Resultados e Discussão}

Para cada linhagem de B. thuringiensis (HD125 e HD1), sequenciou-se um gene vip3Aa. As sequências de nucleotídeos com $2.370 \mathrm{pb}$, que codificaram 789 aminoácidos, foram submetidas ao banco de dados GenBank do NCBI.

A sequência proveniente da linhagem $B$. thuringiensis var. tolworthi HD125 recebeu o número de acesso HQ587048, no GenBank, e foi enviada ao Comitê Bacillus thuringiensis Toxin Gene Nomenclature (Crickmore et al., 2014), para classificação, onde recebeu a denominação de gene vip3Aa42. Verificouse que a sequência de aminoácidos apresentou $99 \%$ de identidade com a sequência do gene vip3Aa35 número de identificação da proteína ADE06071.1 pelo GenBank (2014c), sequenciada a partir do isolado M190 de B. thuringiensis. Duas substituições de bases foram detectadas quando as sequências foram alinhadas com o algoritmo BLASTx, da ferramenta BLAST. As substituições de bases resultaram na substituição dos aminoácidos treonina (vip3Aa35) por alanina (vip3Aa42), no resíduo 484, e glicina (vip3Aa35) por ácido glutâmico (vip3Aa42), no resíduo 659 . 
As substituições dos aminoácidos evidencia a presença de uma nova proteína: Vip3Aa.

O gene vip $3 A a 42$ apresentou identidade de $98 \%$ com a sequência do gene vip3Aal - número de identificação da proteína AAC37036.1 no GenBank (2014d), obtida por Estruch et al. (1996) a partir do isolado AB88. As substituições das bases resultaram na substituição de 12 aminoácidos: glutamina (vip3Aal) por lisina (vip3Aa42), no resíduo 284; isoleucina (vip3Aal) por valina (vip3Aa42), no resíduo 358; treonina (vip3Aal) por alanina (vip3Aa42), no resíduo 484; serina (vip3Aal) por lisina (vip3Aa42), no resíduo 536; asparagina (vip3Aal) por treonina (vip3Aa42), no resíduo 633; glicina (vip3Aal) por ácido glutâmico (vip3Aa42), no resíduo 659; metionina (vip3Aal) por isoleucina (vip3Aa42), no resíduo 755; fenilalanina (vip3Aal) por leucina (vip3Aa42), no resíduo 760; ácido glutâmico (vip3Aal) por glicina (vip3Aa42), no resíduo 761; tirosina (vip3Aal) por asparagina (vip3Aa42), no resíduo 776; histidina (vip3Aal) por lisina (vip3Aa42), no resíduo 782; e tirosina (vip3Aal) por serina (vip3Aa42), no resíduo 784 .

Asequência proveniente da linhagem $B$. thuringiensis var. kurstaki HD1 recebeu o número de acesso HQ594534, no GenBank, e foi enviada ao Comitê Bacillus thuringiensis Toxin Gene Nomenclature (Crickmore et al., 2014), para classificação, onde recebeu a denominação de gene vip3Aa43. Verificouse que a sequência de aminoácidos apresentou $100 \%$ de identidade com 13 sequências classificadas no GenBank-NCBI: Vip3Aa7 - AAK95326.1; Vip3Aa10 - AAN60738.1; Vip3Aa11 - AAR36859.1; Vip3Aa12 - AAM22456; Vip3Aa13 - AAL69542; Vip3Aa15 AAP51131.1; Vip3Aa21 - ABD84410.1; Vip3Aa33 - GU073128; Vip3Aa34 - GU073129; Vip3Aa36 - GU951510; Vip3Aa37 - HM132041; Vip3Aa44 HQ650163; e Vip3Aa55 - KJ868172.

Entre as sequências com $100 \%$ de identidade com o gene vip3Aa43, encontra-se o gene vip $3 A a 33$, cuja sequência também foi obtida a partir da linhagem-padrão B. thuringiensis var. kurstaki HD1, em 2009 (Sauka et al., 2012).

A identidade de $100 \%$ com as 13 sequências depositadas evidencia a presença de alelos iguais aos da classe Vip3 Aa (Crickmore et al., 1998), indício de que as proteínas expressas por estes genes apresentariam o mesmo efeito tóxico quando testadas em uma determinada população, uma vez que são as alterações dos aminoácidos que podem ter efeitos significativos sobre a atividade inseticida e a especificidade, conforme observado por Shen et al. (2009).

O alinhamento entre as sequências de aminoácidos das proteínas Vip3Aa42 e Vip3Aa43 mostrou uma identidade de $99 \%$ entre elas. Vinte e uma substituições de bases foram detectadas no alinhamento com o algoritmo BLASTx. Essas substituições de base resultaram na substituição de 11 aminoácidos: valina (vip3Aa42) por isoleucina (vip3Aa43), no resíduo 358; alanina (vip3Aa42) por treonina (vip3Aa43), no resíduo 484; lisina (vip3Aa42) por serina (vip3Aa43), no resíduo 536; treonina (vip3Aa42) por asparagina (vip3Aa43), no resíduo 633; ácido glutâmico(vip3Aa42) por glicina (vip3Aa43), no resíduo 659; isoleucina (vip3Aa42) por metionina (vip3Aa43), no resíduo 755; leucina (vip3Aa42) por fenilalanina (vip3Aa43), no resíduo 760; glicina (vip3Aa42) por ácido glutâmico (vip3Aa43), no resíduo 761; asparagina (vip3Aa42) por tirosina (vip3Aa43), no resíduo 776; lisina (vip3Aa42) por histidina (vip3Aa43), no resíduo 782; e serina (vip3Aa42) por tirosina (vip3Aa43), no resíduo 784 .

A região N-terminal das proteínas Vip3 é altamente conservada; portanto, as diferenças entre as sequências de aminoácidos encontram-se principalmente na região C-terminal (Wu et al., 2007), e isso também é observado entre as sequências de aminoácidos das proteínas Vip3Aa42 e Vip3Aa43. A diversidade encontrada na família de proteínas Vip é atribuída a mutações que ampliam o espectro de ação inseticida sobre os insetos da ordem Lepidoptera (Wu et al., 2007).

Assim, embora as proteínas Vip3Aa42 e Vip3Aa43 tenham apresentado alta identidade (99\%) entre si, com substituição em apenas 11 aminoácidos, são necessárias análises de sua toxicidade e especificidade para larvas de $S$. frugiperda, $A$. gemmatalis e $H$. virescens, visto que alterações mínimas nas sequências de aminoácidos podem alterá-la, conforme observado com a proteína Vip3Aa (Liu et al., 2007; Shen et al., 2009).

Liu et al. (2007) mostraram que a proteína Vip3Aa19 possui identidade de $97 \%$ com a proteína Vip3Aa11, com diferenças em apenas 19 resíduos de aminoácidos; no entanto, a proteína Vip3Aa19 foi tóxica a S. exigua, Helicoverpa armigera (Hübner, 1808) (Lepidoptera: Noctuidae) e Plutella xylostella (Linnaeus, 1758) (Lepidoptera: Plutellidae), mas foi pouco tóxica a Ostrinia furnacalis (Guenee, 1854) (Lepidoptera: Crambidae); enquanto a proteína Vip3Aa11 foi tóxica 
a $S$. exigua e $H$. armigera, mas apresentou baixa toxicidade a $P$. xylostella.

Shen et al. (2009) realizaram estudos sobre as proteínas Vip3Aa26 e Vip3Aa27, que apresentam identidade de $99 \%$, com diferença em apenas 11 resíduos de aminoácidos, e mostraram que a proteína Vip3Aa27 é altamente tóxica a Trichoplusia $n i$ (Hübner) (Lepidoptera: Noctuidae), S. exigua e $H$. armigera. Em contraste, a proteína Vip3Aa26 apresentou toxicidade apenas para T. ni, o que confirma que alterações mínimas nas sequências de aminoácidos podem alterar o espectro inseticida das proteínas Vip3Aa.

A expressão das proteínas recombinantes foi confirmada pela presença de uma banda de 101,5 kDa, com 88,5 kDa da proteína Vip3Aa e $13 \mathrm{kDa}$ referentes à fusão, na região $\mathrm{N}$-terminal, de um peptídeo com cauda de 6 histidinas e uma proteína Sumo (Figura 1).

Nos bioensaios com os lisados proteicos realizados com $S$. frugiperda, a $\mathrm{CL}_{50}$ estimada foi de 78,2 $\mathrm{ng} \mathrm{cm}^{-2}$,

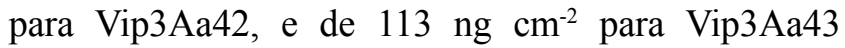
(Tabela 2). Como houve sobreposição dos intervalos de confiança (IC), as proteínas foram consideradas igualmente tóxicas para a espécie. Figueiredo et al. (2013) realizaram ensaios com a proteína Vip3Aa50 e a mesma população de $S$. frugiperda e obtiveram $\mathrm{CL}_{50}$ de 79,6 $\mathrm{ng} \mathrm{cm}^{-2}$. Caccia et al. (2014) relataram toxicidade da proteína Vip3Aa16 testada em neonatas

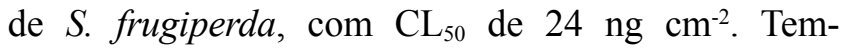
se observado que a proteína Vip3Aa é altamente tóxica a $S$. frugiperda, com toxicidade superior à das proteínas Cry1. Sena et al. (2009) relataram que a proteína Vip3Aa1 apresentou $\mathrm{CL}_{50}$ de $49,3 \mathrm{ng} \mathrm{cm}^{-2}$ para $S$. frugiperda; enquanto as proteínas Cry $1 \mathrm{Ab}$

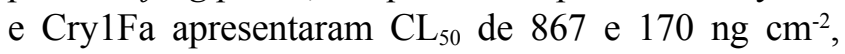
respectivamente. Ao analisar a suscetibilidade de 15 populações de campo de $S$. frugiperda, de diferentes regiões do Brasil, Bernardi et al. (2014) observaram que a proteína Vip3Aa20, introduzida em plantas de milho, apresentou toxicidade entre 92,38 e $611,56 \mathrm{ng} \mathrm{cm}^{-2}$ a essa praga.

Para A. gemmatalis, as proteínas Vip3Aa42 e Vip3Aa43 apresentaram $\mathrm{CL}_{50}$ de 239,2 e $57,5 \mathrm{ng} \mathrm{cm}^{-2}$, respectivamente (Tabela 2). Esse resultado mostra que a proteína Vip3Aa43 é cerca de quatro vezes mais tóxica para essa espécie. Testes realizados por Figueiredo et al. (2013), com a proteína Vip3Aa50, também mostraram que neonatas de A. gemmatalis são altamente suscetíveis às proteínas Vip3Aa, que causaram $\mathrm{CL}_{50}$ de $20,3 \mathrm{ng} \mathrm{cm}^{-2}$. Crialesi-Legori et al. (2014) relataram que as proteínas Vip3Aa, Vip3Ae e Vip3Af têm $\mathrm{CL}_{50}$ de $5,4,5,0$ e $3,6 \mathrm{ng} \mathrm{cm}^{-2}$ para essa espécie, respectivamente. Os autores também relataram alta suscetibilidade da espécie às proteínas Cry1Aa, Cry1Ab, Cry1Ac, Cry1Ca e Cry1Ea.

Pela análise da toxicidade das proteínas Vip3Aa42 e Vip3Aa43, pode-se concluir que a Vip3Aa42 é cerca de três vezes mais efetiva contra $S$. frugiperda do que contra $A$. gemmatalis, em termos de $\mathrm{CL}_{50}$, mas igualmente efetiva em termos de $\mathrm{CL}_{90}$. A proteína Vip3Aa43 foi igualmente efetiva contra $S$. frugiperda e A. gemmatalis, independentemente da concentração letal analisada (Tabela 2).

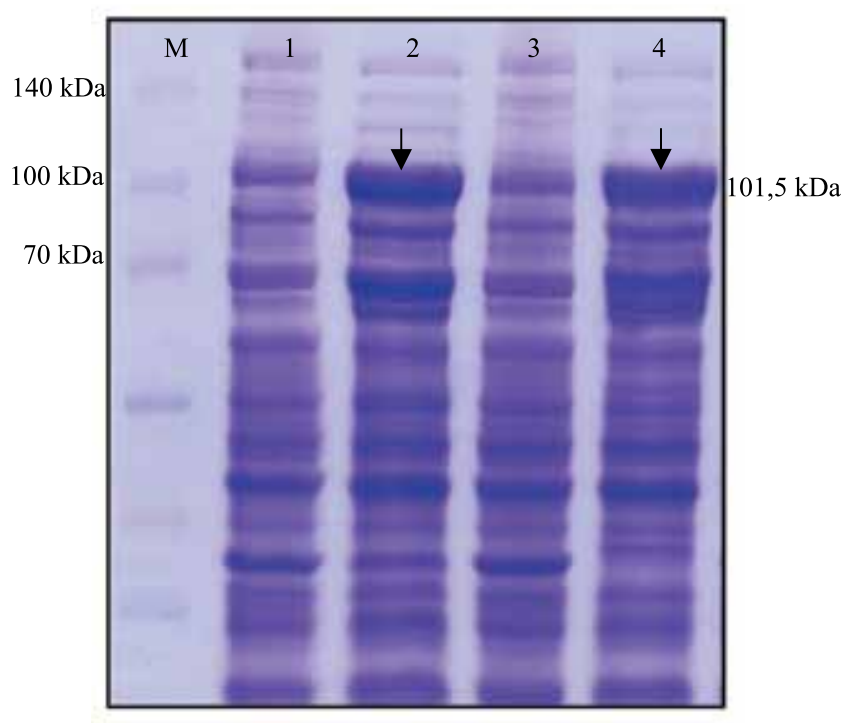

Figura 1. Análise de SDS-PAGE 12\% das proteínas totais dos lisados proteicos de clones de Escherichia coli BL21(DE3), transformados com os genes vip3Aa42 e vip3Aa43. Os genes foram clonados em vetor de expressão pET Sumo, e a expressão foi induzida. M, marcador de massa molecular Spectra Multicolor Broad Range Protein Ladder (Thermo Scientific, Rockford, IL, EUA), em kDa; 1, gene vip3Aa42 não induzido; 2, gene vip3Aa42 induzido; 3, gene vip3Aa43 não induzido; e 4, gene vip3Aa43 induzido. As proteínas recombinantes possuem massa de $101,5 \mathrm{kDa}$ (setas), da qual 88,5 kDa das proteínas Vip3Aa e $13 \mathrm{kDa}$ referentes à fusão, na região $\mathrm{N}$-terminal, de uma cauda com 6 histidinas e uma proteína Sumo, presentes no vetor $\mathrm{pET}$ Sumo. 
As proteínas Vip3Aa42 e Vip3Aa43 apresentaram baixa toxicidade a $H$. virescens, uma vez que não foi possível obter a concentração letal, mesmo em concentrações acima de $5.000 \mathrm{ng} \mathrm{cm}^{-2}$.

Lemes et al. (2014) testaram a toxicidade da proteína Vip3Aa para $H$. virescens e obtiveram $\mathrm{CL}_{50}$ de $1.650 \mathrm{ng} \mathrm{cm}^{-2}$, o que evidencia que esta lagarta de fato apresenta baixa suscetibilidade a proteínas Vip3Aa; no entanto, em comparação a Cry1Aa e Cry $1 \mathrm{Ca}$, a proteína Vip3Aa foi mais efetiva, pois, os autores relataram $\mathrm{CL}_{50}$ estimadas de 3.500 e $3.100 \mathrm{ng} \mathrm{cm}^{-2}$, respectivamente, para essas proteínas. Os autores relatam ainda que a proteína Cry1Ac, com $\mathrm{CL}_{50}$ de $40 \mathrm{ng} \mathrm{cm}^{-2}$, foi cerca de 41 vezes mais tóxica a $H$. virescens do que a Vip3Aa.

Diferenças entre as concentrações letais podem ocorrer também em razão de diferentes fases larvais avaliadas nos bioensaios, e de diferentes metodologias e populações. A diferença entre diferentes populações ocorre em razão da variabilidade genética natural das espécies (Bernardi et al., 2014).

Em relação à ativação proteolítica das proteínas Vip3Aa, que ocorreu pela proteólise de um fragmento com 101,5 $\mathrm{kDa}$ para um de aproximadamente 62 $\mathrm{kDa}$, tanto com uso da tripsina pancreática bovina (Sigma-Aldrich) quanto do suco intestinal dos insetos (Figura 2). Essa ativação foi observada inclusive com o uso do suco intestinal de $H$. virescens, para a qual estas proteínas não foram tóxicas. Portanto, verifica-se que a etapa de ativação não foi a responsável pela ausência de toxicidade para o inseto. Esses resultados foram consoantes com os estudos de ativação da protoxina Vip3A realizados por Lee et al. (2003) com outras espécies de lagartas, e confirmaram que a primeira etapa para a atividade inseticida das proteínas Vip3Aa ocorreu.

Observou-se ligação das proteínas Vip3Aa42 e Vip3Aa43 às VMMAs dos insetos testados (Figura 3). As proteínas Vip3Aa foram tóxicas a $S$. frugiperda e A. gemmatalis, mas não apresentaram toxicidade à população de $H$. virescens testada (Tabela 2). Contudo, houve a ligação de ambas as proteínas nos receptores presentes nas VMMAs também desta última espécie, indício de que a ligação das proteínas aos receptores, apesar de ser um passo necessário para ativação da toxicidade, não é suficiente para garanti-la. O mesmo fato foi relatado por Chakroun \& Ferré (2014), que observaram que a proteína Vip3Ad, embora não tenha sido tóxica a $S$. frugiperda, ligou-se aos receptores na VMMAs.

No ensaio de competição homóloga entre as proteínas, observou-se que a ligação da proteína marcada diminuiu (redução da intensidade da banda), quando se utilizaram 1.000 vezes o excesso de proteína tripsinizada não marcada com biotina. A redução da ligação da proteína marcada tornou-se ainda mais evidente, quando se utilizaram 2.000 vezes o excesso de proteína tripsinizada não marcada, para todas as espécies estudadas. A redução da ligação da proteína marcada ocorre quando os receptores são imediatamente ocupados pelas proteínas não marcadas, utilizadas em excesso.

Análises de competições homólogas, realizadas por Abdelkefi-Mesrati et al. (2011) com a proteína Vip3Aa16, mostraram que esta proteína se liga de maneira específica aos receptores presentes nas VMMAs de Spodoptera littoralis (Boisduval, 1833)

Tabela 2. Concentração letal $\left(\mathrm{CL}_{50} \text { e } \mathrm{CL}_{90}\right)^{(1)}$ das proteínas Vip3Aa42 e Vip3Aa43, em neonatas de Spodoptera frugiperda, Anticarsia gemmatalis e Heliothis virescens.

\begin{tabular}{|c|c|c|c|c|c|c|}
\hline Proteína & $\mathrm{N}$ & $\begin{array}{l}\text { Coeficiente } \\
\text { angular } \pm \mathrm{EP}\end{array}$ & $\mathrm{CL}_{50}$ & $\mathrm{CL}_{90}$ & Qui-quadrado & $\begin{array}{l}\text { Graus de } \\
\text { liberdade }\end{array}$ \\
\hline & \multicolumn{6}{|c|}{ Spodoptera frugiperda } \\
\hline Vip3Aa42 & 640 & $1,2 \pm 0,1$ & $78,2(51,2-128,6)$ & $862,8(401,6-3.563,1)$ & 8,88 & 5 \\
\hline \multirow[t]{2}{*}{ Vip3Aa43 } & 512 & $1,3 \pm 0,1$ & $113,0(86,0-162,3)$ & $977,4(539,8-2.453,3)$ & 3,84 & 4 \\
\hline & \multicolumn{6}{|c|}{ Anticarsia gemmatalis } \\
\hline Vip3Aa42 & 576 & $1,4 \pm 0,1$ & $239,2(172,7-356,7)$ & $1.725,2(952,7-4.564,7)$ & 6,74 & 5 \\
\hline \multirow[t]{2}{*}{ Vip3Aa43 } & 512 & $1,6 \pm 0,1$ & $57,5(36,1-89,1)$ & $338,0(186,2-1.134,1)$ & 8,62 & 4 \\
\hline & \multicolumn{6}{|c|}{ Heliothis virescens } \\
\hline Vip3Aa42 & 512 & - & $>5.000$ & - & - & - \\
\hline Vip3Aa43 & 512 & - & $>5.000$ & - & - & - \\
\hline
\end{tabular}

${ }^{(1)}$ Valores entre parênteses representam o intervalo de confiança, com 95\% de probabilidade. EP, erro-padrão da média. 
(Lepidoptera: Noctuidae), visto que o aumento das quantidades de proteína não marcada (excessos de 50, 100, 400 e 1.000 vezes) reduziu significantemente a ligação da proteína marcada. Segundo Chakroun \& Ferré (2014), a redução da ligação da proteína marcada, quando incubada com excesso de proteína não marcada, confirma que há um número limitado de receptores para Vip3Aa.

Aranda et al. (1996) descreveram que a proteína $\mathrm{Cry} 1 \mathrm{Ab}$ interage de maneira inespecífica com os receptores nas VMMAs de $S$. frugiperda, pois a ligação da toxina marcada não foi afetada pela presença do competidor homólogo em excesso de 300 vezes.

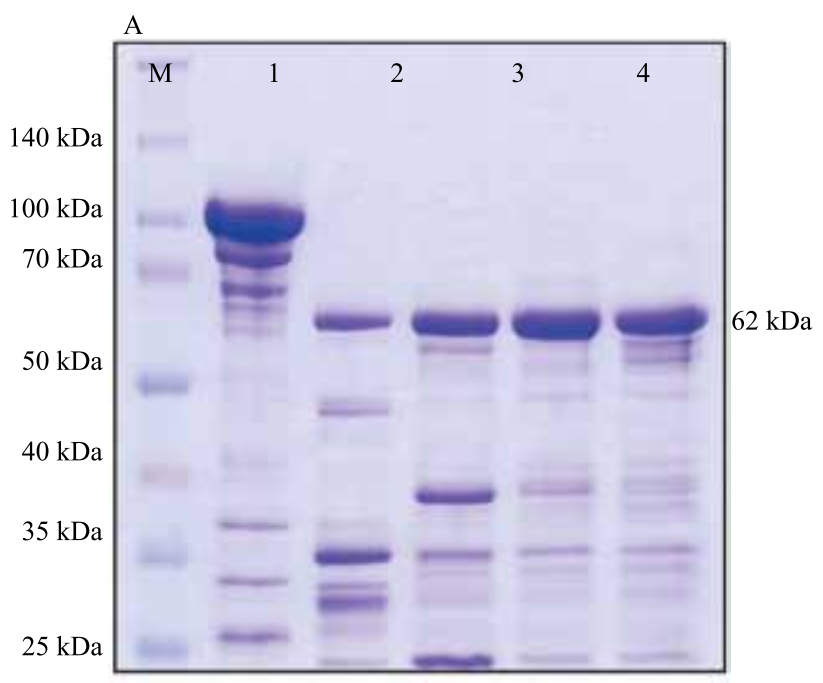

O resultado obtido no presente trabalho, portanto, é indicativo de que existe um grande número de receptores para as proteínas Vip3Aa presentes nas VMMAs dos insetos testados, e que há necessidade de uma alta quantidade (2.000 vezes) de proteína homóloga não marcada, para saturar os sítios receptores, indício de que interações inespecíficas das proteínas Vip3Aa às VMMAs de $S$. frugiperda, A. gemmatalis e H. virescens podem ocorrer.

As proteínas Vip3Aa42 e Vip4Aa43 apresentam grande potencial de controle das espécies $S$. frugiperda e $A$. gemmatalis e podem ser utilizadas efetivamente em estratégias de manejo de resistência dessas pragas.

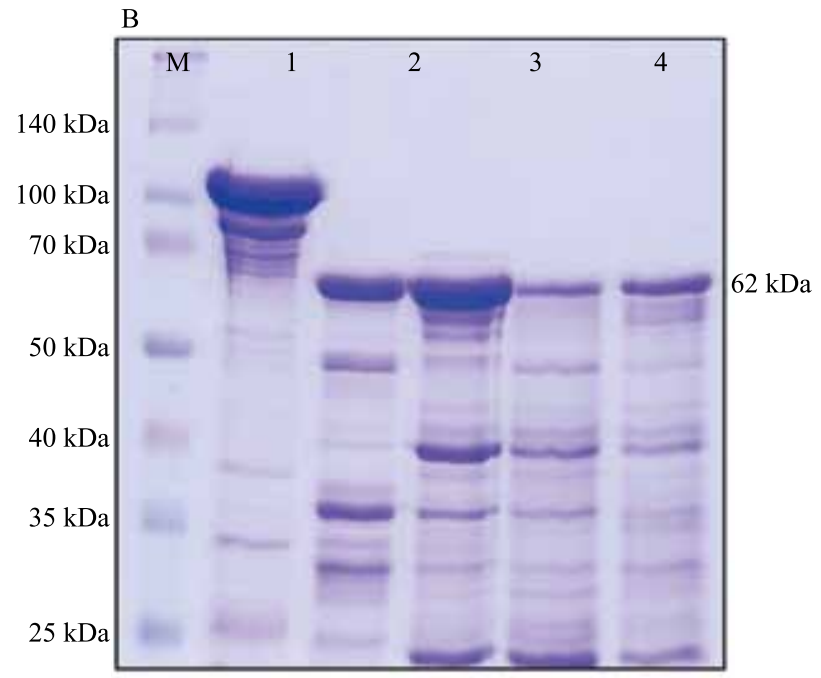

Figura 2. Análise de SDS-PAGE 12\% das proteínas Vip3Aa42 (A) e Vip3Aa43 (B) ativadas. M, marcador de massa molecular Spectra Multicolor Broad Range Protein Ladder (Thermo Scientific), em kDa; 1, proteína purificada; 2, proteína ativada com tripsina pancreática bovina (Sigma- Aldrich); 3, proteína ativada com suco intestinal de Spodoptera frugiperda; 4, proteína ativada com suco intestinal de Anticarsia gemmatalis; e 5, proteína ativada com suco intestinal de Heliothis virescens.
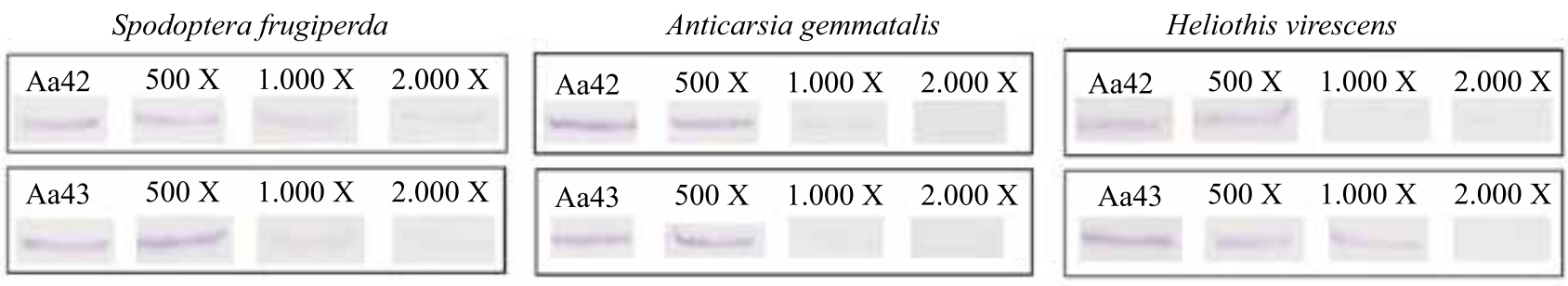

Figura 3. Ensaio de ligação e competição homóloga das proteínas Vip3Aa42 e Vip3Aa43 às vesículas de membrana da microvilosidade apical (VMMAs) de Spodoptera frugiperda, Anticarsia gemmatalis e Heliothis virescens. Aa42, ligação da proteína Vip3Aa42, marcada com biotina, à VMMA; Aa43, ligação da proteína Vip3Aa43, marcada com biotina, à VMMA. Competição homóloga: 500X, 1.000X e 2.000X de proteína não marcada em excesso. 
No Brasil, até o momento, existe apenas um evento transgênico de soja que expressa a proteína CrylAc combinada com a resistência à herbicida (BtRR2Y). Esse evento, apesar de eficiente no controle de A. gemmatalis e Chrysodeixis includens (Walker, 1858) (Lepidoptera: Noctuidae), não é eficiente para o controle de espécies de Spodoptera, que apresentam tolerância natural à proteína Cry1 Ac (Luttrell et al., 1999). Assim, um evento de soja piramidado que expressasse as proteínas Cry1Ac, juntamente com as proteínas Vip3Aa42 ou Vip3Aa43 poderia ser utilizado também para o controle de $S$. frugiperda, que é uma recente praga na cultura da soja no Brasil (Moreira \& Aragão, 2009).

Além disso, os genes vip 3 Aa 42 e vip $3 A a 43$ poderiam ser introduzidos isoladamente, ou em conjunto com genes $c r y$, em genótipos de milho, na busca por plantas resistentes a $S$. frugiperda. No Brasil, há vários eventos de milho-Bt aprovados que expressam diferentes proteínas (Cry1Aa, Cry1Ab, Cry1F e Vip3Aa20); no entanto, S. frugiperda é capaz de evoluir resistência rapidamente, conforme o que se tem observado quanto à toxina Cry1F (Storer et al., 2010; Monnerat et al., 2015). Portanto, o uso das proteínas testadas no presente trabalho também poderia colaborar no manejo de resistência de $S$. frugiperda em milho.

\section{Conclusões}

1. Neonatas de Spodoptera frugiperda e Anticarsia gemmatalis apresentam elevada suscetibilidade a Vip3Aa42 e Vip3Aa43, enquanto neonatas de Heliothis virescens não apresentam suscetibilidade a essas proteínas.

2. As proteínas Vip3Aa42 e Vip3Aa43 se ligam aos receptores presentes nas vesículas de membrana da microvilosidade apical (VMMAs) de $S$. frugiperda, $A$. gemmatalis e $H$. virescens.

3. A capacidade de ligação aos receptores nas VMMAs de $H$. virescens não garante toxicidade das proteínas à espécie.

\section{Agradecimentos}

À Fundação de Amparo à Pesquisa do Estado de São Paulo (Fapesp), pelo apoio financeiro; à Profa. Maria Inês Tiraboschi Ferro e ao Prof. Jesus Aparecido Ferro, Crebio (Centro de Recursos Biológico e Biologia Genômica) da Universidade Estadual Paulista
(Unesp), Campus Jaboticabal, pelo auxílio nas análises das sequências e disponibilização da infraestrutura de bioinformática; ao Prof. José Roberto Postali Parra, do Laboratório de Biologia de Insetos da Escola de Agricultura Luiz de Queiroz (Esalq-USP); e à SGS Gravena Ltda., pelo fornecimento dos insetos utilizados nos bioensaios.

\section{Referências}

ABDELKEFI-MESRATI, L.; BOUKEDI, H.; DAMMAK-KARRAY, M.; SELLAMI BOUDAWARA, T.; JAOUA, S.; TOUNSI, S. Study of the Bacillus thuringiensis Vip3Aa16 histopathological effects and determination of its putative binding proteins in the midgut of Spodoptera littoralis. Journal of Invertebrate Pathology, v.106, p.250-254, 2011. DOI: 10.1016/j.jip.2010.10.002.

ARANDA, E.; SANCHEZ, J.; PEFEROEN, M.; GÜERECA, L.; BRAVO, A. Interactions of Bacillus thuringiensis crystal proteins with the midgut ephitelial cells of Spodoptera frugiperda (Lepidoptera: Noctuidae). Journal of Invertebrate Pathology, v.68, p.203-212, 1996. DOI: 10.1006/jipa.1996.0087.

BERNARDI, O.; AMADO, D.; SOUSA, R.S.; SEGATTI, F.; FATORETTO, J.; BURD, A.D.; OMOTO, C. Baseline susceptibility and monitoring of Brazilian populations of Spodoptera frugiperda (Lepidoptera: Noctuidae) and Diatraea saccharalis (Lepidoptera: Crambidae) to Vip3Aa20 insecticidal protein. Journal of Economic Entomology, v.107, p.781-790, 2014. DOI: 10.1603/ EC13374.

BRADFORD, M.M. A rapid and sensitive method for the quantitation of microgram quantities of protein utilizing the principle of protein-dye binding. Analytical Biochemistry, v.72, p.248-254, 1976. DOI: 10.1016/0003-2697(76)90527-3.

CACCIA, S.; CHAKROUN, M.; VINOKUROV, K.; FERRÉ, J. Proteolytic processing of Bacillus thuringiensis Vip3A proteins by two Spodoptera species. Journal of Insect Physiology, v.67, p.76-84, 2014. DOI: 10.1016/j.jinsphys.2014.06.008.

CHAKROUN, M.; FERRÉ, J. In vivo and in vitro binding of Vip3Aa to Spodoptera frugiperda midgut and characterization of binding sites by ${ }^{125} \mathrm{I}$ radiolabeling. Applied and Environmental Microbiology, v.80, p.6258-6265, 2014. DOI: 10.1128/ AEM.01521-14.

CRIALESI-LEGORI, P.C.B.; DAVOLOS, C.C.; LEMES, A.R.N.; MARUCCI, S.C.; LEMOS, M.V.F.; FERNANDES, O.A.; DESIDÉRIO, J.A. Interação de proteínas Cry1 e Vip3A de Bacillus thuringiensis para controle de lepidópteros-praga. Pesquisa Agropecuária Brasileira, v.49, p.79-87, 2014. DOI: 10.1590/ S0100-204X2014000200001.

CRICKMORE, N.; ZEIGLER, D.R.; FEITELSON, J.; SCHNEPF, E.; VAN RIE, J.; LERECLUS, D.; BAUM, J.; DEAN, D.H. Revision of the nomenclature for the Bacillus thuringiensis pesticidal crystal proteins. Microbiology and Molecular Biology Reviews, v.62, p.807-813, 1998. 
CRICKMORE, N.; BAUM, J.; BRAVO, A.; LERECLUS, D.; NARVA, K.; SAMPSON, K.; SCHNEPF, E.; SUN, M.; ZEIGLER, D.R. Bacillus thuringiensis toxin nomenclature. 2014. Available at: <http://www.btnomenclature.info/>. Accessed on: 5 Nov. 2014.

DUHRUA, S.; GUJAR, G.T. Field-evolved resistance to Bt toxin Cry1Ac in the pink bollworm, Pectinophora gossypiella (Saunders) (Lepidoptera: Gelechiidae), from India. Pest Management Science, v.67, p.898-903, 2011. DOI: 10.1002/ps.2127.

ESTRUCH, J.J.; WARREN, G.W.; MULLINS, M.A.; NYE, G.J.; CRAIG, J.A.; KOZIEL, M.G. Vip3A, a novel Bacillus thuringiensis vegetative insecticidal protein with a wide spectrum of activities against lepidopteran insects. Proceedings of the National Academy of Science United States of America, v.93, p.5389-5394, 1996. DOI: 10.1073/pnas.93.11.5389.

EWING, B.; GREEN, P. Base-calling of automated sequencer traces using phred. II. Error probabilities. Genome Research, v.8, p.186-194, 1998. DOI: 10.1101/gr.8.3.186.

EWING, B.; HILlIER, L.; WENDL, M.C.; GREEN, P. Base-calling of automated sequencer traces using phred. I. Accuracy assessment. Genome Research, v.8, p.175-185, 1998. DOI: $10.1101 /$ gr.8.3.175.

FERRÉ, J.; VAN RIE, J. Biochemistry and genetics of insect resistance to Bacillus thuringiensis. Annual Review of Entomology, v.47, p.501-533, 2002. DOI: 10.1146/annurev. ento.47.091201.145234.

FIGUEIREDO, C.S.; MARUCCI, S.C.; TEZZA, R.I.D.; LEMOS, M.V.F.; DESIDÉRIO, J.A. Caracterização do gene vip3A e toxicidade da proteína Vip3Aa50 à lagarta-do-cartucho e à lagarta-da-soja. Pesquisa Agropecuária Brasileira, v.48, p.1220-1227, 2013. DOI: 10.1590/S0100-204X2013000900005.

FINNEY, D.J. Probit analysis. Cambridge: Cambridge University, 1971. 333p.

GENBANK. Bacillus thuringiensis serovar kurstaki strain HD-1 vegetative insecticidal protein (vip3Aa) gene, complete cds. Available at: <http://www.ncbi.nlm.nih.gov/nuccore/ GU073128>. Accessed on: 5 Nov. 2014a.

GENBANK. Bacillus thuringiensis strain HD125 insecticidal protein Vip3A (vip3A) gene, partial cds. Available at: <http:// www.ncbi.nlm.nih.gov/nuccore/AF399667>. Accessed on: 5 Nov. $2014 b$.

GENBANK. Vip190 [Bacillus thuringiensis]. Available at: $<$ http://www.ncbi.nlm.nih.gov/protein/291465232>. Accessed on: 5 Nov. 2014c.

GENBANK. vip3A(a) [Bacillus thuringiensis]. Available at: $<$ http://www.ncbi.nlm.nih.gov/protein/AAC37036.1>. Accessed on: 5 Nov. 2014 d.

GORDON, D.; ABAJIAN, C.; GREEN, P. Consed: a graphical tool for sequence finishing. Genome Research, v.8, p.195-202, 1998. DOI: $10.1101 /$ gr.8.3.195.

GREENE, G.L.; LEPPLA, N.C.; DICKERSON, W.A. Velvetbean caterpillar: a rearing procedure and artificial medium. Journal of Economic Entomology, v.69, p.487-488, 1976. DOI: 10.1093/ jee/69.4.487.
HAMADOU-CHARFI, D.B.; BOUKEDI, H.; ABDELKEFI-MESRATI, L.; TOUNSI, S.; JAOUA, S. Agrotis segetum midgut putative receptor of Bacillus thuringiensis vegetative insecticidal protein Vip3Aa16 differs from that of Cry1Ac toxin. Journal of Invertebrate Pathology, v.114, p.139-143, 2013. DOI: 10.1016/j.jip.2013.07.003.

HOFMANN, C.; VANDERBRUGGEN, H.; HÖFTE, H.; VAN RIE, J.; JANSENS, S.; VAN MELLAERT, H. Specificity of Bacillus thuringiensis delta-endotoxins is correlated with the presence of high-affinity binding sites in the brush border membrane of target insect midguts. Proceedings of the National Academy of Sciences of the United States of America, v.85, p.7844-7848, 1988. DOI: 10.1073/pnas.85.21.7844.

LAEMMLI, U.K. Cleavage of structural proteins during assembly of the head of bacteriophage T4. Nature, v.227, p.680-681, 1970. DOI: $10.1038 / 227680 \mathrm{a} 0$.

LEE, M.K.; WALTERS, F.S.; HART, H.; PALEKAR, N.; CHEN, J.S. The mode of action of the Bacillus thuringiensis vegetative insecticidal protein Vip3A differs from that of Cry1 Ab delta-endotoxin. Applied and Environmental Microbiology, v.69, p.4648-4657, 2003. DOI: 10.1128/AEM.69.8.4648-4657.2003.

LEMES, A.R.N.; DAVOLOS, C.C.; CRIALESI-LEGORI, P.C.B.; FERNANDES, O.A.; FERRÉ, J.; LEMOS, M.V.F.; DESIDERIO, J.A. Synergism and antagonism between Bacillus thuringiensis Vip3A and Cry1 proteins in Heliothis virescens, Diatraea saccharalis and Spodoptera frugiperda. PLOS One, v.9, p.1-8, 2014. DOI: 10.1371/journal.pone.0107196.

LETOWSKI, J.; BRAVO, A.; BROUSSEAU, R.; MASSON, L. Assessment of cryl gene contents of Bacillus thuringiensis strains by use of DNA microarrays. Applied and Environmental Microbiology, v.71, p.5391-5398, 2005. DOI: 10.1128/ AEM.71.9.5391-5398.2005.

LIU, J.; SONG, F.; ZHANG, J.; LIU, R.; HE, K.; TAN, J.; HUANG, D. Identification of vip3A-type genes from Bacillus thuringiensis strains and characterization of a novel vip3A-type gene. Letters in Applied Microbiology, v.45, p.432-438, 2007. DOI: 10.1111/j.14 72-765X.2007.02217.x.

LOGUERCIO, L.L.; BARRETO, M.L.; ROCHA, T.L.; SANTOS, C.G.; TEIXEIRA, F.F.; PAIVA, E. Combined analysis of supernatant-based feeding bioassays and PCR as a first-tier screening strategy for Vip-derived activities in Bacillus thuringiensis strains effective against tropical fall armyworm. Journal of Applied Microbiology, v.93, p.269-277, 2002. DOI: 10.1046/j.1365-2672.2002.01694.X.

LUTTRELL, R.G.; WAN, L.; KNIGHTEN, K. Variation in susceptibility of noctuid (Lepidoptera) larvae attacking cotton and soybean to purified endotoxin proteins and commercial formulations of Bacillus thuringiensis. Journal of Economic Entomology, v.92, p.21-32, 1999. DOI: 10.1093/jee/92.1.21.

MARÇON, P.C.R.G.; YOUNG, L.J.; STEFFEY, K.L.; SIEGFRIED, B.D. Baseline susceptibility of European corn borer (Lepidoptera: Crambidae) to Bacillus thuringiensis toxins. Journal of Economic Entomology, v.92, p-279-285, 1999.

MONNERAT, R.; MARTINS, E.; MACEDO, C.; QUEIROZ, P.; PRAÇA, L.; SOARES, C.M.; MOREIRA, H.; GRISI, I.; SILVA, J.; 
SOBERÓN, M.; BRAVO, A. Evidence of field-evolved resistance of Spodoptera frugiperda to Bt corn expressing Cry $1 \mathrm{~F}$ in Brazil that is still sensitive to modified Bt toxins. PLOS One, v.10, article number e0119544, 2015. DOI: 10.1371/journal.pone.0119544.

MOREIRA, H.J. da C.; ARAGÃO, F.D. Manual de pragas da soja. Campinas: IAC, 2009. 74p.

RAYMOND, B.; WYRES, K.L.; SHEPPARD, S.K.; ELLIS, R.J.; BONSALL, M.B. Environmental factors determining the epidemiology and population genetic structure of the Bacillus cereus group in the field. PLOS Pathogens, v.6, article number e1000905, 2010.

SAMBROOK, J.; RUSSELL, D.W. Molecular cloning: a laboratory manual. $3^{\text {rd }}$ ed. New York: Cold Spring Harbor, 2001. $250 \mathrm{p}$.

SAUKA, D.H.; RODRIGUEZ, S.E.; BENINTENDE, G.B. New variants of lepidoptericidal toxin genes encoding Bacillus thuringiensis Vip3Aa proteins. Journal of Molecular Microbiology and Biotechnology, v.22, p.373-380, 2012. DOI: 10.1159/000345911.

SENA, J.A.D.; HERNÁNDEZ-RODRÍGUEZ, S.; FERRÉ, J. Interaction of Bacillus thuringiensis Cry1 and Vip3A proteins to Spodoptera frugiperda midgut binding sites. Applied and Environmental Microbiology, v.75, p.2236-2237, 2009. DOI: 10.1128/AEM.02342-08.

SHEN, J.; HOU, M.; GUO, W. Identification and cloning of vip3A genes from isolates of Bacillus thuringiensis and their bioactivity analysis. Wei Sheng Wu Xue Bao, v.49, p.110-116, 2009.

STORER, N.P.; BABCOCK, J.M.; SCHLENZ, M.; MEADE, T.; THOMPSON, G.D.; BING, J.W.; HUCKABA, R.M. Discovery and characterization of field resistance to $\mathrm{Bt}$ maize: Spodoptera frugiperda (Lepidoptera: Noctuidae) in Puerto Rico. Journal of Economic Entomology, v.103, p.1031-1038, 2010. DOI: 10.1603/ EC10040.

TABASHNIK, B.E.; GASSMANN, A.J.; CROWDER, D.W.; CARRIÈRE, Y. Insect resistance to Bt crops: evidence versus theory. Nature Biotechnology, v.26, p.199-202, 2008. DOI: $10.1038 /$ nbt1382.

VACHON, V.; LAPRADE, R.; SCHWARTZ, J.-L. Current models of the mode of action of Bacillus thuringiensis insecticidal crystal proteins: a critical review. Journal of Invertebrate Pathology, v.111, p.1-12, 2012. DOI: 10.1016/j.jip.2012.05.001.

VAN FRANKENHUYZEN, K. Insecticidal activity of Bacillus thuringiensis crystal proteins. Journal of Invertebrate Pathology, v.101, p.1-16, 2009. DOI: 10.1016/j.jip.2009.02.009.

WOLFERSBERGER, M.; LUETHY, P.; MAURER, A.; PARENTI, P.; SACCHI, F.V.; GIORDANA, B.; HANOZET, G.M. Preparation and partial characterization of amino acid transporting brush border membrane vesicle from the larval midgut of the cabbage butterfly (Pieris brassicae). Comparative Biochemistry and Physiology. Part A: Physiology, v.86, p.301-308, 1987. DOI: 10.1016/0300-9629(87)90334-3.

WOLFERSBERGER, M.G. The toxicity of two Bacillus thuringiensis $\delta$-endotoxins to gypsy moth larvae is inversely related to the affinity of binding sites on midgut brush border membranes for the toxins. Experientia, v.46, p.475-477, 1990. DOI: 10.1007/ BF01954236.

WU, J.; ZHAO, F.; BAI, J.; DENG, G.; QIN, S.; BAO, Q. Evidence for positive Darwinian selection of vip gene in Bacillus thuringiensis. Journal of Genetics and Genomics, v.34, p.649-660, 2007. DOI: 10.1016/S1673-8527(07)60074-5.

Recebido em 27 de fevereiro de 2015 e aprovado em 25 de junho de 2015 